\title{
Temporal Bone Metastasis of Follicular Thyroid Carcinoma in an Asymptomatic Patient: A Rare Case
}

\author{
Ahmad Khairi Mohamed $^{1}$, Irfan Mohamad ${ }^{1}$, Norazlina Mat Nawi², Mohd Fazrin \\ Mohd Rohani
}

${ }^{1}$ Department of Otorhinolaryngology - Head \& Neck Surgery, School of Medical Sciences, Universiti Sains Malaysia Health Campus, Kubang Kerian, Kelantan, Malaysia; ${ }^{2}$ Department of Nuclear Medicine, Radiotherapy \& Oncology, School of Medical Sciences, Universiti Sains Malaysia Health Campus, Kubang Kerian, Kelantan, Malaysia

\begin{abstract}
Introduction: Thyroid carcinomas are classified according to their histological subtypes, namely: papillary thyroid carcinoma (PTC), follicular thyroid carcinoma (FTC), medullary carcinoma, undifferentiated carcinoma and poorly differentiated carcinoma. PTC usually spread to the lymph node which can be the presenting complaint, whereas FTC tends to have distant metastasis owing to its propensity of hematogenous spread. Differentiated thyroid carcinomas (DTC) such as papillary thyroid carcinoma (PTC) and follicular thyroid carcinoma (FTC) have a better prognosis compare to medullary and undifferentiated types. FTC constitutes around 10 to $25 \%$ of thyroid malignancies. Despite of its rarity compared to PTC, FTC tends to have distant metastases to lungs and bones. Usually long bones such as femur are more affected. Metastases to the skull and skull base are rare with only few reported cases.
\end{abstract}

We report a case of temporal bone metastasis in an asymptomatic follicular thyroid carcinoma, which was detected on Iodine-131 ( $\left.{ }^{131} \mathrm{I}\right)$ scan and characterized on single-photon emission computed tomography/ computed tomography (SPECT/CT) scan.

Conclusion: Temporal bone is a rare metastatic site of FTC. Early detection and prompt treatment play a vital role in patients' survival and reducing the metastatic risk. In post total thyroidectomy patients, post ablation ${ }^{131} \mathrm{I}$ whole body scan with SPECT/CT and serum thyroglobulin correlation is essential in detection of distant metastasis in asymptomatic patients.

Keywords: Follicular Thyroid Carcinoma; Temporal Bone; Metastasis; Radioiodine; Singlephoton emission computed tomography 